\title{
EDUCAÇÃO A DISTÂNCIA NA PÓS-GRADUAÇÃO LATO SENSU \\ NA ÁREA DA SAÚDE: uma revisão integrativa
}

\section{E-LEARNING IN HEALTH POST-GRADUATION: an integrative review}

\author{
Gabriela Eyng Possolli ${ }^{1}$ \\ Débora Maria Vargas Makuch ${ }^{2}$ \\ Eliane Rozados Fernandes Costa ${ }^{3}$ \\ Salete Terezinha Arcanjo ${ }^{4}$
}

\section{Resumo}

A evolução das tecnologias de informação e comunicação possibilitou o desenvolvimento da Educação a Distância (EAD) em instituições de Educação Superior ampliando o acesso à formação na pós-graduação. Para estabelecer as relações entre EAD e a formação dos profissionais da saúde no Brasil e no mundo, optou-se pela revisão integrativa objetivando compreender sobre o que versam as publicações entre 2009 e 2014 relacionadas à EAD na pós-graduação, em português, inglês e espanhol. Aplicados os critérios de inclusão e exclusão e mapeando-se os artigos disponíveis resultou um grupo relevante de artigos analisados em três categorias: 1- EAD na área da saúde na pós-graduação; 2- Processo de implantação e desenvolvimento de cursos de pós-graduação lato sensu na área da saúde a distância; 3 Desafios e Potencialidades da pós-graduação a distância na área da saúde. Como resultado, além da análise das categorias, conclui-se que artigos relevantes foram publicados e demonstraram a consolidação da EAD como modalidade pertinente e de qualidade na formação de profissionais da saúde na pós-graduação.

Palavras-chave: educação a distância (EAD). pós-graduação lato sensu. instituições de educação superior (IES). tecnologias de informação e comunicação (TICs).

\begin{abstract}
The expansion of information and communication technologies providing the development of e-learning in universities, extending the access in higher education. To establish the relations between e-learning and health professionals development in Brazil and the world, it was opted for an integrative review defining as main objective to understand what the publications between 2009 and 2014 related to the e-learning in the post-graduation, in Portuguese, English and Spanish. Applied inclusion and exclusion criteria and studies analysis resulting in 12 papers and three categories: 1- e-learning in health post-graduation; 2- implementation of health post- graduation in the e-learning modality; 3- challenges and potentialities of health post-graduation through e-learning. One concludes that excellent papers had been published and demonstrated the consolidation of the e-learning as pertinent modality and of quality in the development of health professionals' post-graduation.
\end{abstract}

Key-words: e-learning. post-graduation. Universities. information and communication

\footnotetext{
${ }^{1}$ A autora é Doutora em Educação (UFPR) e Coordenadora de educação a distância da Faculdades Pequeno Príncipe (FPP). E-mail: gabriela.possolli@fpp.edu.br

${ }^{2}$ A autora é Mestranda em Ensino nas ciências da saúde (FPP) e Professora dos cursos de graduação da Faculdades Pequeno Príncipe (FPP). E-mail: deboramakuch@ hotmail.com

3 A autora é Mestranda em Ensino nas ciências da saúde (FPP); Licenciada em Pedagogia (FALEC); coordenadora de pós-graduação da Faculdades Pequeno Príncipe (FPP). E-mail: eliane.costa@fpp.edu.br

4 A autora é Bacharel em Biblioteconomia (UEL), pós-graduada em gestão empresarial (FACESI) e Coordenadora da biblioteca da Faculdades Pequeno Príncipe (FPP). E-mail: salete.arcanjo@fpp.edu.br
} 
technology (ICT).

\section{INTRODUÇÃO}

A efetivação da educação a distância como modalidade para implementação de ações na Educação Superior se deu com ênfase nas últimas duas décadas embasada fortemente em tecnologias de informação e comunicação. As indagações e interesses de pesquisa que moveram a condução do grupo para a produção desse artigo de análise integrativa estão relacionados aos perceptíveis avanços das tecnologias de informação e comunicação nas últimas décadas que afetaram a existência humana em todas as áreas de conhecimento. Esses avanços estabelecem campos de pesquisa para que o pensamento educacional ocupe-se das possibilidades, limitações e rumos que essas tecnologias apresentam à contemporaneidade, sobretudo, no que se refere à educação a distância no nível de pós-graduação. Nessa conjuntura de desenvolvimento vertiginoso de tecnologias digitais, notável a partir dos anos 2000, a educação a distância regulamentada por um conjunto de políticas e normativas governamentais, surgiu nas instituições de Educação Superior, que anteriormente ocupavam-se somente de ações de ensino, pesquisa e extensão presenciais, como uma nova modalidade de ensino-aprendizagem.

O desenvolvimento da EAD em instituições de Educação Superior públicas e privadas relaciona-se diretamente com a evolução das tecnologias de informação e comunicação que possibilitaram a criação de novas formas de: acompanhamento e mediação pedagógica, progressos nos mecanismos de interatividade e ampliação da oferta de oportunidades de formação em nível superior. O estabelecimento de políticas, definindo diretrizes para a aplicação de ações de educação a distância nas IES, tendo como pano de fundo as TIC, e focando especificamente a formação de profissionais da saúde na pós-graduação, constitui um campo a ser desbravado e entendido em profundidade. A relevância educacional e científica da presente revisão integrativa reside na atualidade da proposta e na necessidade crescente de se realizar pesquisas atualizadas visando a estabelecer relações entre a modalidade de educação a distância e a formação dos profissionais da saúde no Brasil e no mundo.

Para a efetivação deste estudo optou-se pela revisão integrativa, sendo suas etapas desenvolvidas conforme referencial de Souza, Silva e Carvalho (2010) e as cinco fases por eles propostas. O objetivo estruturante dessa revisão integrativa foi: compreender sobre o que versam as publicações dos últimos seis anos relacionadas à educação a distância na pósgraduação nas principais bases de dados acessadas pela comunidade brasileira nos idiomas português, inglês e espanhol. Esse estudo se posiciona como uma contribuição que destaca a 
necessidade (confirmada por essa pesquisa) de elevação da quantidade de pesquisas publicadas que divulguem as experiências institucionais com EAD na pós-graduação e embasem teoricamente tais ações.

\section{DISCUSSÃO}

\subsection{PRIMEIRA FASE: ELABORAÇÃO DA QUESTÃO NORTEADORA}

Levando-se em conta a realidade referente ao acesso dos profissionais da saúde à educação superior e à posterior especialização em áreas de seu interesse, bem como, a crescente conectividade às diferentes mídias, elaborou-se a seguinte questão norteadora: Sobre o que versam as publicações relacionadas à educação a distância na pós-graduação?

Por meio dessa questão objetivou-se compreender como artigos que versam sobre ações a distância na pós-graduação desenvolvem a temática, elencando categorias e subtemas trabalhados e detalhando a abrangência dada ao tema na literatura a fim de compreender os avanços científicos nessa área.

\subsection{SEGUNDA FASE: BUSCA OU AMOSTRAGEM NA LITERATURA}

Os critérios de inclusão a este estudo foram: artigos na íntegra e disponíveis nos idiomas português, espanhol e inglês, no período de janeiro de 2009 a dezembro de 2014, e relacionados ao tema. Como critérios de exclusão: teses e dissertações, artigos em outros idiomas que não o português, espanhol e inglês, período anterior a 2009 e posterior a 2014, redundâncias e conteúdos não relacionados ao tema, bem como artigos pagos.

\subsection{TERCEIRA FASE: COLETA DE DADOS}

A busca de artigos foi realizada no mês de dezembro de 2014 na Biblioteca Virtual em Saúde (BVS) que é um Centro Especializado da Organização Pan-Americana da Saúde (OPAS) estabelecido no Brasil desde 1967, que congrega as bases de dados: Literatura LatinoAmericana e do Caribe em Ciências da Saúde (LILACS), Índice Bibliográfico Espanhol de Ciências da Saúde (IBECS), Literatura Internacional em Ciências da Saúde (MEDLINE), Biblioteca Cochrane e Scientific Eletronic Library Online (SciELo). Para a busca de artigos foram utilizados os descritores abrangentes: educação a distância e pós-graduação. Obteve-se um total de 439 publicações, dessas: 130 artigos disponíveis, 113 artigos na íntegra, 78 no período estabelecido, 77 nos idiomas selecionados, 54 artigos versando sobre os temas 
educação a distância e pós-graduação. Esses 54 artigos foram avaliados pelas quatro pesquisadoras integrantes da equipe de pesquisa. Todas analisaram o resumo de cada um dos artigos avaliando se este deveria ou não integrar a amostra a ser utilizada. Foram realizadas reuniões para que os avaliadores compartilhassem suas avaliações. Após uma nova análise em grupo, foram selecionados aqueles que de fato abordavam a discussão de temáticas relacionadas à pós-graduação e educação a distância ou tecnologias vinculadas a essa modalidade, tendo sido aprovados 23 artigos para a próxima fase de análise, que foi a leitura dos artigos na íntegra e o levantamento de categorias. O Quadro 1 apresenta os artigos selecionados para leitura na íntegra em um processo de duplo cego, em que duas pessoas leram o mesmo artigo buscando aprová-lo para a fase final e extrair categorias de análise para encontrar os pontos de convergência entre os textos dos vários autores. Nesse Quadro é possível visualizar o título dos artigos, o assunto central e a decisão (especificando o motivo de exclusão de alguns deles para a fase final):

\section{Quadro 1 - Dados dos artigos selecionados para $2^{\text {a }}$ fase: título, assunto e decisão}

\begin{tabular}{|c|c|c|c|}
\hline \begin{tabular}{|c|}
$2 a$ \\
Fase
\end{tabular} & Título do Artigo & Assunto do Artigo & Decisão após leitura na íntegra \\
\hline 1 & $\begin{array}{l}\text { Panorama da educação a distância em } \\
\text { enfermagem no Brasil }\end{array}$ & $\begin{array}{l}9 \text { cursos de pós-EAD na área de } \\
\text { enfermagem; EAD na formação de } \\
\text { enfermeiros }\end{array}$ & $\begin{array}{l}\text { Aprovado para fase de análise de } \\
\text { categorias }\end{array}$ \\
\hline 2 & $\begin{array}{l}\text { Challenges and opportunities in graduate } \\
\text { nursing education by distributed learning in } \\
\text { Canada and Brazil }\end{array}$ & $\begin{array}{l}\text { Cooperação Canadá Brasil e oferta de pós- } \\
\text { EAD para enfermagem }\end{array}$ & $\begin{array}{l}\text { Aprovado para fase de análise de } \\
\text { categorias }\end{array}$ \\
\hline 3 & $\begin{array}{l}\text { Webinar software: a tool for developing } \\
\text { more effective lectures (online or in-person) }\end{array}$ & $\begin{array}{l}\text { Seminário online na residência médica (61 } \\
\text { seminários em } 1 \text { ano) }\end{array}$ & $\begin{array}{l}\text { Aprovado para fase de análise de } \\
\text { categorias }\end{array}$ \\
\hline 4 & $\begin{array}{l}\text { Twelve tips on how to set up postgraduate } \\
\text { training via remote clinical supervision }\end{array}$ & $\begin{array}{l}\text { Supervisão remota de médicos residentes } \\
\text { por médicos especializados }\end{array}$ & $\begin{array}{l}\text { Excluído - disponível somente } \\
\text { resumo, para acessar artigo } \\
\text { completo é necessário pagar. }\end{array}$ \\
\hline 5 & $\begin{array}{l}\text { Teaching physiology online: successful use } \\
\text { of case studies in a graduate course }\end{array}$ & $\begin{array}{l}\text { Curso de pós-graduação on-line em } \\
\text { fisiologia usando estudos de caso }\end{array}$ & $\begin{array}{l}\text { Aprovado para fase de análise de } \\
\text { categorias }\end{array}$ \\
\hline 6 & $\begin{array}{l}\text { Kenya and distance education: a model to } \\
\text { advance graduate nursing }\end{array}$ & $\begin{array}{l}\text { Pós-graduação a distancia para enfermeiros no } \\
\text { Quênia }\end{array}$ & $\begin{array}{l}\text { Aprovado para fase de análise de } \\
\text { categorias }\end{array}$ \\
\hline 7 & $\begin{array}{l}\text { Current trends in systematic program } \\
\text { evaluation of online graduate nursing } \\
\text { education: an integrative literature review }\end{array}$ & $\begin{array}{l}\text { Avaliação de programas de educação de } \\
\text { enfermagem on-line em nível de pós- } \\
\text { graduação }\end{array}$ & $\begin{array}{l}\text { Excluído - disponível somente } \\
\text { resumo, para acessar artigo } \\
\text { completo é necessário pagar. }\end{array}$ \\
\hline 8 & $\begin{array}{l}\text { Aspectos relativos a la estancia de los } \\
\text { estudiantes universitarios y de pos } \\
\text { graduación en disciplinas semipresenciales }\end{array}$ & $\begin{array}{l}\text { Pós-graduandos em disciplinas } \\
\text { semipresenciais da área da saúde }\end{array}$ & $\begin{array}{l}\text { Aprovado para fase de análise de } \\
\text { categorias }\end{array}$ \\
\hline 9 & $\begin{array}{l}\text { Planning a graduate programme in public } \\
\text { health nutrition for experienced nutrition } \\
\text { professionals }\end{array}$ & $\begin{array}{l}\text { Modelo de planejamento THCU para um } \\
\text { programa de pós-graduação em nutrição em } \\
\text { saúde pública }\end{array}$ & $\begin{array}{l}\text { Aprovado para fase de análise de } \\
\text { categorias }\end{array}$ \\
\hline 10 & $\begin{array}{l}\text { Study on the feasibility of provision of } \\
\text { distance learning programmes in surgery to } \\
\text { Malawi }\end{array}$ & $\begin{array}{l}\text { Viabilidade da prestação de curso a } \\
\text { distância em cirurgia no Malawi-Africa - } \\
\text { alto interesse em pós-EAD, mas a } \\
\text { infraestrutura é ruim (acesso caro e lento) }\end{array}$ & $\begin{array}{l}\text { Aprovado para fase de análise de } \\
\text { categorias }\end{array}$ \\
\hline
\end{tabular}




\begin{tabular}{|c|c|c|c|}
\hline 11 & $\begin{array}{l}\text { The Great Plains IDEA Gerontology } \\
\text { Program: an online, interinstitutional } \\
\text { graduate degree }\end{array}$ & $\begin{array}{l}\text { Programa de pós-graduação em } \\
\text { Gerontologia - avaliação realizada em sete } \\
\text { estados nos EUA para determinar o } \\
\text { interesse em um programa de pós- } \\
\text { graduação on-line em Gerontologia } \\
\end{array}$ & $\begin{array}{l}\text { Excluído - disponível somente } \\
\text { resumo, para acessar artigo } \\
\text { completo é necessário pagar. }\end{array}$ \\
\hline 12 & $\begin{array}{l}\text { Academic performance in a } \\
\text { pharmacotherapeutics course sequence } \\
\text { taught synchronously on two campuses } \\
\text { using distance education technology }\end{array}$ & $\begin{array}{l}\text { Curso de farmacoterapia - no final do artigo } \\
\text { fala sobre comparação de desempenho } \\
\text { presencial e EAD }\end{array}$ & $\begin{array}{l}\text { Aprovado para fase de análise de } \\
\text { categorias }\end{array}$ \\
\hline 13 & $\begin{array}{l}\text { Teaching medical professionals online: a } \\
\text { cross-discipline experience }\end{array}$ & $\begin{array}{l}\text { Mestrado on-line no programa de educação } \\
\text { - Seis categorias emergiram dos } \\
\text { comentários dos formadores sobre o ensino } \\
\text { on-line para profissionais de saúde em } \\
\text { comparação com o presencial }\end{array}$ & $\begin{array}{l}\text { Excluído - disponível somente } \\
\text { resumo, para acessar artigo } \\
\text { completo é necessário pagar. }\end{array}$ \\
\hline 14 & $\begin{array}{l}\text { Implementation of the virtual learning } \\
\text { environment into a UK orthodontic training } \\
\text { programme: the postgraduate and lecturer } \\
\text { perspective }\end{array}$ & $\begin{array}{l}\text { AVA como apoio ao ensino didático da } \\
\text { pós-graduação ortodôntica }\end{array}$ & nálise de \\
\hline 15 & $\begin{array}{l}\text { An evaluation of the effects of a web-based } \\
\text { modular teaching programme, housed } \\
\text { within a virtual learning environment on } \\
\text { orthodontic training for specialist registrars }\end{array}$ & $\begin{array}{l}\text { Ensino modular via AVA para } \\
\text { complementar a formação acadêmica de } \\
\text { pós-graduação em ortodontia }\end{array}$ & $\begin{array}{l}\text { Excluído - disponível somente } \\
\text { resumo, para acessar artigo } \\
\text { completo é necessário pagar. }\end{array}$ \\
\hline 16 & $\begin{array}{l}\text { essos de ensino- } \\
\text { ções educacionais }\end{array}$ & $\begin{array}{l}\text { Avaliação da aprendizagem - pós-EAD e } \\
\text { grad semipresencial }\end{array}$ & $\begin{array}{l}\text { lo para fase de análise de } \\
\text { s }\end{array}$ \\
\hline 17 & $\begin{array}{l}\text { Lessons learned: providing access through } \\
\text { distance education }\end{array}$ & $\begin{array}{l}\text { Transformação de pós-graduação de } \\
\text { enfermagem em saúde mental } 20 \text { anos } \\
\text { presencial em EAD }\end{array}$ & $\begin{array}{l}\text { Excluído - não é artigo, é um } \\
\text { depoimento de cerca de } 30 \text { linhas }\end{array}$ \\
\hline 18 & $\begin{array}{l}\text { The coordinator role for a nursing online } \\
\text { graduate practicum }\end{array}$ & $\begin{array}{l}\text { Concepção e implementação de } \\
\text { coordenadoção de estágio em um programa } \\
\text { de pós-graduação on-line }\end{array}$ & $\begin{array}{l}\text { Excluído - disponível somente } \\
\text { resumo, para acessar artigo } \\
\text { completo é necessário pagar. }\end{array}$ \\
\hline 19 & $\begin{array}{l}\text { Telementoring: use of augmented reality in } \\
\text { orthopaedic education: AAOS exhibit } \\
\text { selection }\end{array}$ & $\begin{array}{l}\text { Avaliar a eficiência e desempenho de um } \\
\text { sistema VIP (presença virtual) } \\
\text { implementado em centro cirúrgico }\end{array}$ & $\begin{array}{l}\text { Excluído - não aborda } \\
\text { diretamente a pós-graduação }\end{array}$ \\
\hline 20 & $\begin{array}{l}\text { A pilot project in distance education: nurse } \\
\text { practitio-ner students' experience of personal } \\
\text { video capture technology as an assessment } \\
\text { method of clinical skills }\end{array}$ & $\begin{array}{l}\text { Experiências de uso de uma tecnologia de } \\
\text { captura de vídeo pessoal para completar } \\
\text { avaliações de competência }\end{array}$ & $\begin{array}{l}\text { Excluído - fala sobre gravação de } \\
\text { vídeo para exame físico não } \\
\text { abordando diretamente a pós- } \\
\text { graduação }\end{array}$ \\
\hline 21 & $\begin{array}{l}\text { Is obstetrics and gynaecology going the } \\
\text { distance? }\end{array}$ & $\begin{array}{l}\text { EAD para residência em Obstetrícia e } \\
\text { Ginecologia: tecnologias interativas, } \\
\text { opiniões e novas perspectivas }\end{array}$ & $\begin{array}{l}\text { Excluído - a modalidade é } \\
\text { graduação e não pós }\end{array}$ \\
\hline 22 & $\begin{array}{l}\text { Web-based course in neuroimmune } \\
\text { pharmacology }\end{array}$ & $\begin{array}{l}\text { Módulos de aprendizagem na web junto com } \\
\text { palestras face-a-face na área de farmacologia } \\
\text { neuroimune }\end{array}$ & $\begin{array}{l}\text { Aprovado para fase de análise de } \\
\text { categorias }\end{array}$ \\
\hline 23 & $\begin{array}{l}\text { Web-based faculty development: e-learning } \\
\text { for clinical teachers in the London Deanery }\end{array}$ & $\begin{array}{l}\text { Desenvolvimento profissional dos } \\
\text { professores e supervisores clínicos de pós- } \\
\text { graduação via EAD }\end{array}$ & $\begin{array}{l}\text { Excluído - disponível somente } \\
\text { resumo, para acessar artigo } \\
\text { completo é necessário pagar. }\end{array}$ \\
\hline
\end{tabular}

Fonte: elaboração própria / pesquisa de revisão integrativa

Após essa fase ficaram 12 artigos para fase final de análise das categorias. Com relação ao ano de publicação e local/revistas em que foram publicados segue detalhamento: os 12 artigos selecionados estão localizados na série histórica de 2009 a 2014, sendo que sete artigos são dos anos de 2011 e 2012, dois do ano de 2009 e três dos anos de 2013 e 2014. Quanto ao idioma nove artigos estão em inglês, dois em português e um em espanhol. Quatro 
artigos foram publicados em revistas brasileiras da região Sul e Sudeste (Rev Esc Enferm USP, Revista Gaúcha de Enfermagem, Acta Paulista de Enfermagem, Temas em Psicologia) e os outros oito em revistas internacionais (Middle East African Journal of Ophthalmology, Adv Physiol Educ, International Journal of Nursing Practice, Public Health Nutr, The Surgeon, American Journal of Pharmaceutical Education, Eur J Dent Educ, J Neuroimmune Pharmaco).

\subsection{QUARTA FASE: ANÁLISE CRÍTICA DOS ESTUDOS INCLUÍDOS}

O Gráfico 1 demonstra a classificação dos artigos analisados com relação ao tipo de metodologia utilizada. Os tipos encontrados foram: pesquisa descritiva, exploratória, explanatória, relato de experiência; e abordagem: quantitativa, qualitativa e quanti-quali.

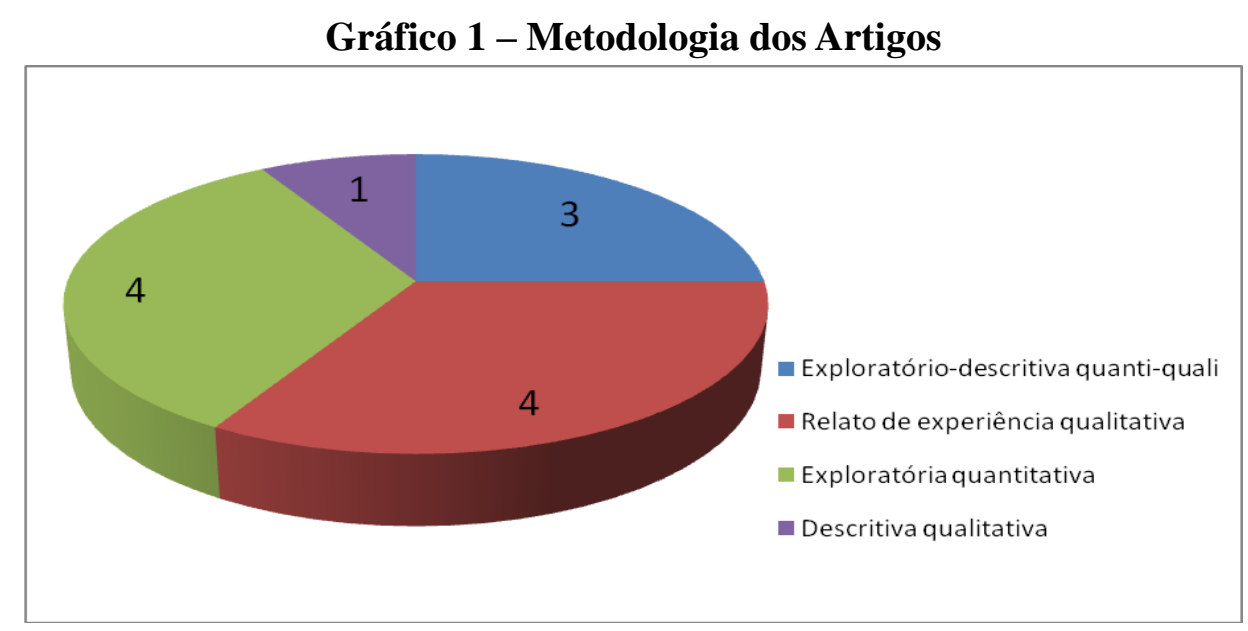

Fonte: elaboração própria / pesquisa de revisão integrativa

Em alguns artigos a metodologia estava explicitada, outros foram analisados pelo grupo de pesquisa e classificados com relação ao tipo de pesquisa e abordagem. Ficou evidente o equilíbrio entre as metodologias encontradas e a prevalência de artigos que detalham experiências institucionais de desenvolvimento de cursos de pós-graduação a distância ou disciplina/módulos a distância mediante abordagem qualitativa.

Durante as leituras cada avaliador elencou categorias e as descreveu, preenchendo uma ficha de avaliação produzida para padronizar as análises e sistematizar as informações encontradas. Após o preenchimento das fichas de avaliação para cada artigo aprovado, para a última fase formam levantadas categorias que foram discutidas em várias reuniões com o grupo de pesquisa. As categorias encontradas foram congregadas em três grupos mais genéricos para se chegar aos itens de análise comuns aos 12 artigos (Quadro 2): 
Quadro 2 - Descrição das categorias emergentes da análise dos artigos

\begin{tabular}{|l|l|}
\hline Categoria & Descrição da Categoria \\
\hline $\begin{array}{l}\text { Educação a distância (EAD) na área } \\
\text { da Saúde na pós-graduação }\end{array}$ & $\begin{array}{l}\text { Mapeamento com objetivo de identificar cursos de pós-graduação na área da } \\
\text { saúde que comportem ações a distância no âmbito mundial e a evolução da } \\
\text { EAD }\end{array}$ \\
\hline $\begin{array}{l}\text { Processo de implantação e } \\
\text { desenvolvimento de cursos de pós- } \\
\text { graduação lato sensu na área da saúde } \\
\text { na modalidade a distância }\end{array}$ & $\begin{array}{l}\text { Relaciona elementos para implantação de cursos na modalidade a distância, } \\
\text { apontando aspectos favoráveis e primordiais apoiados em tecnologias de } \\
\text { comunicação e informação destacando o planejamento estratégico } \\
\text { (Cooperação interinstitucional, Infraestrutura para EAD), qualificação em } \\
\text { EAD (preparo técnico-científico e ético, mídias/comunicação, papel do } \\
\text { professor/Instrutor ou mentor), produção de material didático (casos clínicos } \\
\text { discutidos, atividades, material do curso) e processo avaliativo. }\end{array}$ \\
\hline $\begin{array}{l}\text { Desafios e Potencialidades da pós- } \\
\text { graduação a distância na área da } \\
\text { saúde. }\end{array}$ & $\begin{array}{l}\text { Descrição das Potencialidades da educação a distância identificadas e } \\
\text { detalhadas nos artigos (expansão da EAD, novas oportunidades de formação } \\
\text { permanente, flexibilidade, pós-graduação na Enfermagem, contribuições da } \\
\text { EAD para a educação médica, parcerias entre instituições, motivação para } \\
\text { estuar a distância, superação da dicotomia entre as modalidades presencial e a } \\
\text { distância) e Desafios (capacitação de pessoal, tutoria com qualidade, } \\
\text { feedback, evasão, inclusão digital). }\end{array}$ \\
\hline
\end{tabular}

Fonte: elaboração própria / pesquisa de revisão integrativa.

\subsection{QUINTA-FASE: DISCUSSÃO DOS RESULTADOS}

\section{Categoria I - Educação a Distância (EAD) na área da saúde na pós-graduação}

A primeira categoria que se pretendia verificar com a revisão integrativa é o panorama atual da pós-graduação na modalidade a distância na área da saúde e que tipo de cursos vêm sendo ofertados. Com relação ao panorama atual esse objetivo não foi cumprido como esperado, pois a intensão dos artigos existentes não foi fazer um mapeamento com objetivo de identificar cursos de ensino na área da saúde na modalidade educação a distância no âmbito mundial estabelecendo uma relação com a evolução da EAD. Realizando a leitura dos artigos, no entanto, foi possível fazer uma aproximação desse objetivo, por meio do levantamento de alguns dados e estabelecendo análises sobre realidade atual da EAD no Brasil e as aplicação para a EAD na pós-graduação em saúde em diversos países.

O censo 2013 da Associação Brasileira de Educação a Distância (ABED) foi citado em um dos artigos, apontando que nesse ano existiam mais de 3,5 milhões de alunos matriculados na modalidade a distância. Considerando os cursos formais, autorizados pelo Ministério da Educação, a marca está próxima de 800 mil matrículas, sendo quase $20 \%$ na pós-graduação. (ABED, 2013). 
Os cursos de pós-graduação a distância precisam ser autorizados pelo Ministério da Educação, mas há bastante liberdade na adoção da metodologia. O que o Ministério da Educação exige é que as provas sejam realizadas nos polos de apoio presencial e que a defesa do trabalho de conclusão de curso também seja presencial e síncrona com uma banca de professores que avalia, mesmo que a distância, interagindo com o aluno.

A rotina básica de estudos ocorre com os alunos estudando por meio de ambientes virtuais online, com material multimídia (composto por textos, vídeos, animações e atividades interativas). Os cursos também precisam contar com tutores, que são professores que ficam à disposição para tirar dúvidas dos alunos. Há cursos baseados em telessalas, com realização de transmissão de aulas via satélite, enquanto outros cursos têm todo o material postado online e o aluno progride conforme o ritmo que lhe convier dentro do cronograma estipulado. Esse é um modelo compatível com o que também é realizado na Europa e nos Estados Unidos, o que foi observado em algumas descrições operacionais em certos pontos dos artigos.

Existem boas opções de pós-graduação à distância na rede pública. A Universidade Aberta do Brasil (UAB) oferece alguns cursos, com prioridade para a formação continuada de professores e funcionalismo público, mas outras pessoas também podem se candidatar a esses cursos mediante uma reserva de vagas para o público em geral. As especializações são dadas por universidades públicas de todo o Brasil com reconhecida qualidade. A UAB foi citada de maneira rápida em dois artigos (ROJO, 2011; COMIN, 2013) lidos ao longo do processo de revisão integrativa. A UAB oferece, por exemplo, uma pós-graduação lato sensu, com 360 horas de duração, para formar gestores públicos com a participação de polos em todo o Brasil. A UAB também é responsável pelo primeiro curso de pós-graduação stricto sensu semipresencial do Brasil, um programa de Mestrado Profissional em Matemática em Rede Nacional.

Do mesmo modo que as IES particulares, a UAB mantém polos regionais de apoio presencial. Os polos são mantidos pelas prefeituras ou governos estaduais que possuem convênio com o sistema UAB e oferecem infraestrutura tecnológica e pedagógica para os alunos e servem como locais para encontros presenciais e avaliações. O processo de seleção para ingresso dos alunos é feito pelas instituições públicas de ensino superior que fazem parte do sistema. A UAB foi criada pelo governo federal em 2005 e também oferece cursos de graduação, sendo que a pós-graduação representa atualmente 35\% das matrículas. (POSSOLLI, 2012). 
Dentre os 12 artigos analisados integralmente vários apresentaram relatos de experiências de criação de cursos, disciplinas/módulos de pós-graduação a distância e avaliação de desempenho dos alunos nesses cursos, bem como análises com orientações para viabilidade de ações que incorporem recursos da EAD. A seguir são detalhadas as principais contribuições com relação aos tipos de ações em EAD e indicação dos resultados obtidos segundo exposto nos artigos:

- Artigo de revisão de 2011 apresentando o modelo de funcionamento de nove cursos de pós-graduação a distância na área de enfermagem. Houve a comprovação de vantagens superior às desvantagens na formação continuada de enfermeiros nessa modalidade e indicou-se a expansão de oferta de cursos a distância para qualificação de enfermeiros em serviço rompendo barreiras de tempo e espaço existentes no Brasil. (ROJO, 2011).

- Relato dos desafios e oportunidades em uma parceria entre instituição brasileira com instituição canadense na condução de cursos de pós-graduação na área de enfermagem. Esse artigo de 2009 posiciona que os estudantes avaliam positivamente a experiência e esforços tem sido feitos para assegurar, de modo inovador e flexível, a excelência de programas de pós-graduação em enfermagem a distância. Os autores argumentam que apesar dos desafios existentes, os cursos oferecem alta qualidade e atendem às necessidades de muitos enfermeiros. (MOLZAHN et al, 2009).

- Relatos de experiências na utilização da EAD em residência médica: dois artigos abordam o uso de tecnologias a distância como suporte para residências médicas presenciais. O primeiro, de 2014, relata a experiências de realização de dezenas de seminários online durante dois anos para que os residentes estivessem em contato com professores e profissionais de outros locais para palestras e estudos de caso. O outro, de 2011, investigou os requisitos de usuário, requisitos técnicos e conexões de internet em dois hospitais de ensino no Malawi buscando a vinculação desses hospitais com hospitais universitários de outros países visando a estabelecer parcerias para aprimorar os conhecimentos oferecidos aos residentes. (MAYORGA; BEKERMAN; PALLIS, 2014; MAINS et al., 2011).

- Cinco artigos abordaram os impactos da realização de disciplinas/módulos a distância comparativamente ao presencial demonstrando resultados positivos:

$\checkmark$ O primeiro, publicado em 2013, traz orientações sobre como ensinar fisiologia online estruturando a disciplina com metodologias ativas em um curso de pós-graduação. 
$20 \%$ dos alunos tinham experiência anterior com EAD, porém todos afirmaram que recomendariam o curso para outras pessoas. A maioria dos alunos referiu que aprendeu mais conteúdo utilizando a literatura do que em uma aula tradicional. Os resultados indicam que um curso de fisiologia bem organizado usando um formato de estudo de caso é um modelo eficaz para a aprendizagem online. (CASOTTI; BENESKI; KNABB, 2013);

$\checkmark$ O segundo, de 2012, analisa os aspectos relacionados com a permanência de estudantes de pós-graduação em disciplinas semipresenciais. Foram aplicados vários instrumentos de avaliação de desempenho dos estudantes, concluindo-se que o uso do ambiente virtual, a flexibilidade do tempo e do espaço, os custos envolvidos, o uso das ferramentas computacionais e os procedimentos de interação foram as principais vantagens da aprendizagem a distância que contribuíram para um ótimo desempenho não deixando a desejar com relação à modalidade presencial (PEIXOTO; PEIXOTO; ALVES, 2012);

$\checkmark$ O terceiro, de 2011, analisa a performance acadêmica em cursos em fármacoterapêuticos ensinados em sincronia em dois campi usando tecnologias de educação a distância. Foi comparado o desempenho acadêmico dos estudantes presenciais em um curso de fármaco-terapêuticos com os de estudantes da mesma disciplina a distância (ensinados por meio de teleconferência síncrona). Os escores médios de exames e notas finais dos alunos ao longo de cinco anos não foram significativamente diferentes entre os alunos presenciais e a distância. $\mathrm{O}$ uso das tecnologias de educação a distância síncrona para ensinar os alunos não afeta o desempenho acadêmico, quando usado em um aprendizado ativo, baseado em casos e interação online. (STEINBERG; MORIN, 2011);

$\checkmark$ O quarto artigo, de 2013, versou sobre a avaliação dos processos de ensinoaprendizagem em ações educacionais ofertadas a distância. $O$ foco estava em verificar como ocorria a avaliação da aprendizagem e suas repercussões. $O$ predomínio foi de avaliação baseada em testes somativos aplicados em momentos isolados do curso, como testes automatizados, com geração de feedback. Como conclusão foi exposto que as ações educacionais devem considerar não apenas o produto final como atividade avaliativa, mas fundamentalmente o processo norteador da atividade, seus diferentes passos, desafios, insights e construções colaborativas nos diferentes ambientes virtuais de aprendizagem. (COMIN, 2013);

$\checkmark$ O quinto e último, de 2011, aborda um módulo web em farmacologia neuroimune que combina módulos de aprendizagem online com palestras tradicionais face-a-face. 
O conteúdo do curso se mostrou apropriado para estudantes de pós-graduação. Além de fornecer um plano de estudos, o artigo descreve o processo de criação dos módulos baseados na web para a construção dos conteúdos. (MCMILLAN, 2011).

- A implementação do ambiente virtual de aprendizagem em um programa de ortodontia no Reino Unido segundo a perspectiva do pós-graduando e do professor foi o tema do artigo publicado em 2009. Entrevistas indicaram que o coordenador do programa, os professores e a maioria dos pós-graduandos estavam confiantes de que o AVA contribuiu para produzir uma melhor experiência de aprendizagem, com um grande potencial para incentivar uma abordagem construtivista de ensino-aprendizagem. (SHAH; CUNNINGHAM, 2009).

- Artigo que descreve o planejamento de um programa de pós-graduação para profissionais de nutrição experientes foi publicado em 2011. Segundo a argumentação desenvolvida a renovação da saúde pública no Canadá destacou a necessidade de desenvolvimento e expansão dos profissionais da nutrição na saúde pública, principalmente em comunidades do norte e rurais. A partir dessa contextualização é proposto um planejamento detalhado para que o curso seja desenvolvido. (FOX; BEYERS, 2011).

- Um artigo de 2012 abordou a educação a distância como uma modalidade promissora para o avanço da pós-graduação de enfermagem no Quênia contribuindo para a superação do isolamento de algumas comunidades e intercâmbio de saberes com regiões da África mais avançadas. No Quênia não há oportunidades significativas aos enfermeiros para buscarem pós-graduação devido ao apoio limitado do governo e do sistema de ensino existente. Embora o ensino a distância esteja disponível no Quênia para profissionais como professores, nas universidades públicas, esse tipo de oportunidade é inacessível para os enfermeiros que estão trabalhando e precisam continuar sua formação. Um modelo colaborativo a distância é apresentado como uma solução potencial para essa necessidade com a participação de quatro principais constituintes: hospitais e agências de saúde, comunidades de interesse, universidades do Quênia e parceiros internacionais de educação. (MUTAE; CULLEN, 2012).

\section{Categoria II - Processo de Implantação e Desenvolvimento de cursos de pós-graduação lato sensu na área da saúde na modalidade a distância}

O projeto de implantação de um curso a distância contempla o processo de ensino e aprendizagem por meio do alinhamento da organização curricular aos objetivos de 
aprendizagem, da seleção de equipe multidisciplinar qualificada para esta modalidade, do preparo do material didático com antecedência (pelo menos seis meses antes do início do curso), da promoção da interação de alunos e professores, da utilização de métodos de avaliação de ensino e aprendizagem abrangentes, além de uma infraestrutura de apoio, gestão consciente e delineamento de custos. (ROJO et al. 2011; SHAH; CUNNINGHAM, 2009; CASOTTI; BENESKI; KNABB, 2013; MUTEA; CULLEN, 2012; MAYORGA, BEKERMAN; PALIS, 2014).

Mutea e Cullen (2012) ao abordar a dificuldade na especialização de profissionais de enfermagem no Quênia destacam a EAD como uma possível solução para o treinamento clínico e a educação profissional permanente, mas devido aos custos elevados da implantação desta modalidade para a pós-graduação, refere ser fundamental a colaboração genuína entre comunidade, universidade, instituição de saúde e empresas privadas.

Proporcionar um ambiente educacional seja presencial ou a distância requer conhecimento com relação à forma como o indivíduo aprende, desenvolve habilidades e capacidades cognitivas sociais, emocionais, profissionais e éticas e, em especial, como os adultos aprendem. Suas motivações dependem da sua demanda diária, da implementação prática daquela informação na resolução de problemas e da apresentação do contexto real de aplicação deste conhecimento. (MAYORGA; BEKERMAN; PALIS, 2014; ROJO et al. 2011). Mayorga, Bekerman e Palis (2014, p. 125) relatam que,

\footnotetext{
os princípios que orientam o desenvolvimento do processo educacional para adultos indicam o uso de técnicas de aprendizado baseado na experiência caracterizadas pela possibilidade de aplicação prática, ser instigante, racional, participativo, tendo objetivos claramente definidos, definindo o contexto de vida diário e pactuando feedback formativo.
}

De acordo com Shah e Cunningham (2009), existem diversas teorias e modelos de ensino-aprendizagem por meio da tecnologia, dentre eles, o Mayes Conceptualisation Cycle and Laurillard's Conversational Model baseado na teoria construtivista de Vygotsky. Mayes identificou o processo de conceituação, construção e dialógica no aprendizado com tecnologia. A efetividade do modelo heurístico em que o Ambiente Virtual de Aprendizagem (AVA) é baseado vai depender do contexto em que ele será utilizado sendo um desafio pedagógico explorar novos métodos de ensino. A promoção de oficinas nas quais os docentes irão testar as ferramentas disponíveis experienciando diretamente os recursos em tecnologia. Avaliações formativas, debates síncronos e assíncronos, seminários tutoriais (SHAH; CUNNINGHAM, 2009; CASOTTI; BENESKI; KNABB, 2013; MAYORGA; BEKERMAN; PALIS, 2014). 
Casotti, Beneski e Knabb (2013, p. 66) afirmam que,

ensinar em um curso online pode ser intimidante se, acreditar que toda e qualquer nova ferramenta tecnológica deva ser utilizada. Estar preparado para aprender novas tecnologias porém, mantendo a simplicidade e as necessidades de seus alunos como prioridade.

Nesse sentido, uma equipe de facilitadores com experiência em ensino online é valiosa no desenvolvimento de um curso na modalidade EAD, pois oferecem ideias e norteiam a organização dos materiais. (CASOTTI; BENESKI; KNABB, 2013; MAYORGA, BEKERMAN; PALIS, 2014).

Rojo et al. (2011), Shah e Cunningham (2009), Casotti, Beneski e Knabb (2013), Mayorga, Bekerman e Palis (2014) referem-se à utilização de módulos com estudos de caso de acordo com os assuntos de interesse dos estudantes e com os objetivos de aprendizagem, proporcionando a interação, o reconhecimento e o respeito a diferentes culturas e formas de construção do conhecimento. Rojo et al. (2011) ressalta a importância do feedback e do desenvolvimento de um mix de atividades alinhadas aos casos, em sequência lógica, sendo compromisso do instrutor responder às perguntas e dúvidas dos alunos em até 48 horas após publicação online.

Avaliações formativas e somativas são importantes para garantir o sucesso do modelo implementado. Avaliações formativas devem ser realizadas no decorrer do curso por professores e estudantes em relação a critérios previamente estabelecidos e fazendo com que o próprio estudante seja o protagonista de seu aprendizado (ROJO et al. 2011; SHAH; CUNNINGHAM, 2009; CASOTTI; BENESKI; KNABB, 2013; MUTEA; CULLEN; 2012; MAYORGA; BEKERMAN; PALIS, 2014).

\section{Categoria III - Desafios e Potencialidades da pós-graduação a distância na área da saúde}

A EAD, assim como qualquer modalidade nova de educação que se embasa em tecnologias em processos de democratização e que exigem adaptações pessoais e organizacionais, torna-se uma opção até pouco tempo polêmica. Na última década, com a ampliação das políticas educacionais, a criação da Universidade Aberta do Brasil (UAB) e adesão dessa modalidade com ações de apoio ao presencial e cursos semipresenciais por IES nacionais e internacionais de tradição centenária, a EAD consolidou o seu espaço e comprovou a sua relevância. Assim, desafios e potencialidades são identificados e os artigos 
retratam os principais deles sistematizados na produção acadêmica dos últimos seis anos. Os desafios identificados nos artigos podem ser agrupados em cinco tópicos:

- Capacitação de pessoal: é necessário que seus atores estejam devidamente preparados em relação aos aspectos técnico-científicos e éticos que envolvem esse fazer pedagógico.

- Tutoria com qualidade: uma vez que o contato tutor/aluno foi estabelecido, é essencial definir uma comunicação regular flexível e efetiva. A disponibilidade da equipe de tutoria é a pedra angular para a aprendizagem do aluno (via telefone, webcam, fórum, chat ou e-mail). Para a eficácia dos programas de EAD em nível micro deve-se considerar a integração com uma proposta macro que uma IES tem para o trabalho com TIC. Isso garantirá o suporte adequado para a execução de programas com relação ao apoio para os estudantes. Iniciativas de capacitação específica para EAD são importantes e preconizadas por organismos governamentais.

- Feedback: como os alunos ficam distribuídos em polos em diversas localidades, a previsão e a agilidade dos feedbacks dados por professores e tutores é fundamental para a vinculação, motivação e permanência dos alunos nos cursos.

- Evasão: aspectos que precisam ter o foco da instituição para minimizar o risco da evasão que é maior na EAD: satisfação com o ambiente do curso (serviços disponíveis, serviço rápido às necessidades do aluno, interatividade, ambiente e material); habilidade e competência relacionada com o uso de ferramentas tecnológicas (fórum, chat, mensagens, e-mail); contexto social do aluno (renda familiar, moradia, família, trabalho e outros) e; interação entre os atores envolvidos no processo educativo.

- Inclusão Digital: o acesso à internet e uso do computador estão crescendo no Brasil e em todo o mundo, no entanto, eles ainda não estão disponíveis para todos, o que limita as oportunidades para os indivíduos e os grupos sociais que ainda não desenvolveram as competências e habilidades necessárias, ou simplesmente não têm os recursos apropriados. No Brasil, cerca de $40 \%$ da população ou nunca usou a internet ou não têm acesso regular ou não conseguem entender corretamente a informação. Por outro lado, determinar a exclusão digital ou inclusão simplesmente por ter um computador em casa com ligação à internet é uma simplificação, uma avaliação superficial do problema.

Com relação às potencialidades da EAD na pós-graduação na área de saúde, se destacam os seguintes pontos: 
- Expansão da EAD: a expansão dessa modalidade de ensino por meio de uma série de políticas de regulamentação e incentivo tem viabilizado o acesso à educação superior na graduação e na pós-graduação, democratizando as oportunidades de acesso e permanência na academia.

- Novas oportunidades de formação permanente: a EAD com qualidade proporciona condições favoráveis à formação/aprimoramento dos profissionais da área da saúde, em consonância com a demanda do mercado de trabalho por profissionais capacitados e com domínio em seu campo de atuação sem longos períodos de afastamento dos seus postos de trabalho e com a possibilidade de intercambio interinstitucional.

- Flexibilidade: as tecnologias digitais disponíveis em ambientes online permitem que os alunos e professores encaixem seus estudos no contexto de suas vidas (trabalho, casa, família), minimizando a necessidade de deslocamentos e rompendo com as limitações de tempo e espaço para ensinar e aprender. Nesse item se encaixa ainda a possibilidade de adaptação curricular com base nas necessidades de determinadas instituições, regiões ou perfis de alunos.

- Pós-graduação na Enfermagem: enfermeiros desejam acesso à educação prática avançada para equipá-los com o conhecimento relevante para enfrentar e resolver os problemas de saúde complexos que surgem na gestão e no cuidado dos pacientes. Vários artigos analisados abordam a pós-graduação em enfermagem a distância, apresentando benefícios para os alunos, permitindo que se engajem em programas educacionais que de outra forma seriam incapazes de completar. Esses artigos abordam o desenvolvimento contínuo do corpo docente, a orientação interativa e personalizada aos alunos, o desenvolvimento de políticas e acompanhamento para melhorar continuamente as ações a distância.

- Contribuições da EAD para a educação médica: conclui-se com os relatos que a EAD possibilita a educação médica no mundo em desenvolvimento que tem recursos e oportunidades limitadas. Com a expansão do acesso à internet e computadores há o potencial de treinar estudantes por programas EAD.

- Parcerias entre instituições: alguns artigos apresentam a EAD como uma possibilidade promissora e eficiente para parcerias interinstitucionais para o desenvolvimento de programas de pós-graduação. Um dos artigos, por exemplo, cita um consórcio entre hospitais e agências, comunidades de interesse, universidades do Quênia e parceiros internacionais compondo uma rede integrada de educação.

- Motivação para estudar a distância: os principais aspectos de permanência na EAD segundo os artigos analisados são: o uso de computadores em diferentes atividades; 
conciliação da classe com outras atividades de estudo e compromissos familiares; a disponibilidade do computador; o material do curso disponível online, manutenção de computadores e acesso à Internet; conciliação das atividades profissionais; acessar o curso na internet e conteúdo de estudo com a regularidade pretendida; a qualidade da conexão de internet e mensagens administrativas enviadas; uso de mensagens, fóruns e chats para comunicação com os tutores e colegas.

- Superação da dicotomia entre as modalidades presencial e a distância: discute a partir dos dados anteriormente apresentados que o desempenho dos alunos não foi inferior, e sim que não houve diferença significativa. Os resultados representam a flexibilidade dos estudantes para aprender em uma arena nova e adaptarem-se a maneiras não tradicionais de conduzir o processo de construção do conhecimento.

\section{CONSIDERAÇÕES FINAIS}

Quando um aluno se propõe a realizar uma pós-graduação a distância é a flexibilidade que mais o atrai, uma vez que os profissionais da saúde encontram dificuldade para dispor de momentos presenciais para dedicação a sua formação. Em uma pós-graduação tradicional é preciso se deslocar algumas vezes por semana até a instituição de ensino para assistir às aulas, nos cursos a distância o aluno estuda em casa, no escritório e ou em qualquer tempo e local em que disponha de um dispositivo com acesso à internet, porém é necessário que se discipline para não procrastinar as atividades que precisam ser feitas em cada módulo/disciplina do curso. A questão financeira também costuma pesar na escolha, já que o curso a distância geralmente é mais barato para o aluno em função das características do processo, havendo ainda uma maior mobilidade no que diz respeito ao uso do tempo no curso. Essa modalidade também é bastante indicada para profissionais que precisam se especializar, mas viajam muito e não querem assistir aulas aos finais de semana.

Apesar da liberdade e flexibilidade, é justamente por causa delas, como destacado em alguns dos artigos analisados, que é preciso haver muita disciplina para que os alunos acompanhem bem uma pós-graduação a distância. Há maior flexibilidade de horários, mas os cursos a distância têm uma programação que deve ser seguida. Essa programação não só é um calendário indicativo de como o aluno deve estudar, mas prevê ainda datas específicas em que existem encontros para fazer atividades online com o tutor e colegas, como chats e discussão em fórum, e períodos para assistir aulas. É algo extremamente organizado e, por isso, requer disciplina do aluno. 
Mesmo contando com a dedicação do estudante e da equipe pedagógica outra questão que frequentemente é levantada, abordada também em vários dos artigos que participaram da revisão integrativa, diz respeito à qualidade de um curso de pós-graduação a distância em comparação com um curso presencial. Via de regra, as instituições que têm qualidade no presencial também costumam ter qualidade na educação a distância. Para comprovar isso, alguns dos artigos aqui estudados fizeram análises comparativas de cursos inteiros e disciplinas/módulos presenciais e a distância comprovando que as iniciativas a distância não deixam nada a desejar e ainda apresentam vantagens impactantes em alguns casos em que as barreiras de tempo e espaço se tornam intransponíveis para ações presenciais de formação na área de saúde.

Uma questão que dificultou a realização desse artigo de revisão foi a prática internacional, sobretudo, dos Estados Unidos, adotada por muitas revistas de cobrar o acesso aos artigos. Encontramos um valor médio de U\$35 para acesso durante espaços curtos de tempo e geralmente sem a possibilidade de baixar os arquivos. Tal realidade dificulta o acesso ao conhecimento e o avanço da ciência uma vez que os resultados de pesquisas não ficam acessíveis para a grande maioria das pessoas, perdendo assim o sentido de publicar resultados de pesquisas que serão vistos por poucos.

Um dos artigos trouxe uma contribuição muito pertinente com relação ao processo de planejamento para um programa de pós-graduação a distância, sistematizando um modelo que é interessante constar como contribuição nesse artigo de revisão. Trata-se de um modelo de planejamento de um programa desenvolvido com a integração de vários departamentos da área de saúde de uma grande IES canadense. Esse modelo propõe quatro etapas: 1- Pré-planejamento e gestão de projetos: aborda o modo como o processo de planejamento acontecerá. Durante essa fase, as partes interessadas são identificadas e passam a discutir como irão trabalhar em conjunto, como os conhecimentos serão construídos e como serão tomadas as decisões. 2- Avaliação situacional: envolve a avaliação da situação atual, incluindo necessidades, lacunas e oportunidades, bem como os pontos fortes e os recursos existentes. Esta fase tenta confirmar ou não se o planejamento deve prosseguir. 3- Identificar metas, populações de interesse e objetivos / identificar estratégias, atividades, recursos e indicadores de desenvolvimento: esculpir as metas e objetivos, estratégias e atividades e os resultados pretendidos. Isso inclui a identificação de indicadores que serão usados para acompanhar o progresso. 4- Revisão do plano de programa: criação de metas, objetivos e estratégias para os indicadores, a fim de apoiar a avaliação do programa. A retroalimentação é incorporada em cada passo do processo. Embora 
cada passo seja representado separadamente no modelo, algumas situações de planejamento requerem mais do que um passo e podem acontecer simultaneamente ou em diferentes sequências. Depois do planejamento vêm a implementação e os resultados/impactos.

A falta de conhecimento sobre as características de um curso a distância ainda provoca certa discriminação no mercado. Já para o Ministério da Educação, a validade dos cursos presenciais e a distância é a mesma, sendo que os cursos a distância devem seguir normativas especiais e passam por avaliação específica para estarem credenciados. Cabe frisar que no certificado emitido após a conclusão do curso não existe diferenciação entre cursos presenciais e a distância.

A educação a distância deve continuar se expandindo nos próximos anos com a tendência de que a difusão da modalidade caminhe junto com o desenvolvimento das tecnologias digitais. Além disso, a facilidade de acesso e de uso dessas tecnologias também permite a ampliação da oferta desses cursos. Para os profissionais da saúde interessados em cursar uma pós-graduação a distância a principal questão a ser analisada, além de se certificar de que a instituição tem autorização do Ministério da Educação para oferecer a pós-graduação a distância, está em conhecer o modelo de EAD proposto e material didático para saber se a metodologia utilizada possui a qualidade necessária.

A revisão integrativa hora realizada demonstrou que nos últimos seis anos artigos relevantes foram publicados e avançaram na sistematização de experiências e demonstraram a consolidação da EAD como modalidade pertinente e de qualidade para a formação de profissionais da saúde na pós-graduação. Há muito ainda a ser pesquisado e divulgado para que a comunidade acadêmica compreenda melhor os modelos de EAD existentes e como operacionalizar cursos a distância com o uso das tecnologias de informação e comunicação que estão em plena evolução.

\section{REFERÊNCIAS}

ABED. Associação Brasileira de Educação a Distância. Censo EAD.BR: Relatório Analítico da Aprendizagem a Distância no Brasil 2012. Curitiba, PR: Ibpex, 2013.

CASOTTI, G.; BENESKI, J.T.; KNABB, M.T. Teaching physiology online: successful use of case studies in a graduate course. Adv Physiol Educ., v. 37, p. 65-69, 2013.

COMIN, F. S. Avaliação dos processos de ensino-aprendizagem em ações educacionais ofertadas a distância. Temas de psicologia, v. 21, n. 2, p. 335-346, dez. 2013.

FOX, A; CULLEN, D. Kenya and distance eduction: a model to advance graudate nursing. Int 
J Nurs Prac..; v. 18, n. 4, p. 417-22, aug. 2012.

MAINS, E. A et al. Study on the feasibility of provision of distance learning programmes in surgery to Malawi. The Surgeon, v.9, n. 6, p. 322-5, dec. 2011.

MAYORGA, E. P.; BEKERMAN, J. G.; PALIS, A.G. Webinar Software: A Tool for Developing More Effective Lectures (Online or In-Person). Middle East African Journal of Ophthalmology, v. 21, n.2, p.123-127, 2014

MCMILLAN, D. C. Web-based Course in Neuroimmune Pharmacology. Jorunal of Neuroimmune Pharmacology. v.6, n.1, p.76-79, march 2011.

MOLZAHN, A. (et al.). Challenges and oppontunities in graduate nursing education by distributed learning in Candada and Brazil. Revista gaúcha de enfermagem. v 30, n 4, p.755761, dez., 2009.

MUTEA, N; CULLEN, D. Kenya and distance education: A model to advance graduate nursing. International Journal of Nursing Practice, v.18, p.417-422, 2012.

PEIXOTO, H. M.; PEIXOTO, M. M.; ALVES, E. D. Aspectos related to the permancence of undergraduate and graduate students in semi-presential classes. Acta Paulista de Enfermagem. São Paulo, v. 25, n. 2, p. 48-53, 2012.

POSSOLLI, G. E. Políticas de Educação Superior a distância e os pressupostos para formação de professores. Tese (Doutorado em Educação) - Universidade Federal do Paraná, Curitiba, 2012.

ROJO, P. T. et al. Panorama da educação a distância em enfermagem no Brasil. Revista da Escola de Enfermagem da USP, v. 45, n.6, p.1476-1480, dez./2011.

SHAH, R; CUNNINGHAM, S.J. Implementation of the virtual learning environment into a UK orthodontic training programme: the postgraduate and lecturer perspective. Eur J Dent Educ., v.13, p.223-232, 2009.

SOUZA, M. T.; SILVA, M. D.; CARVALHO, R. Revisão integrativa: o que é e como fazer. Einstein, v.8, n.1, p. 102-106, 2010.

STEINBERG, Morin; MORIN A. K. Academic performance in a pharmacotherapeutics course sequence taught synchronously on two campuses using distance education technology. Am J Pharm Educ., v. 75, n. 8, p. 15, oct.2010. 